\title{
Liver Abscess Caused by Multidrug-resistant Pseudomonas aeruginosa Treated with Colistin; A Case Report and Review of the Literature
}

\author{
Kenichiro Yaita ${ }^{1,4}$, Ichiro Sameshima ${ }^{2}$, Hideaki Takeyama ${ }^{3}$, Shinpei Matsuyama ${ }^{3}$, \\ Chie Nagahara ${ }^{4}$, Ryo Hashiguchi ${ }^{4}$, Yukiko Moronaga ${ }^{4}$, Nami Tottori ${ }^{4}$, Masanari Komatsu ${ }^{1,4}$, \\ Yusuke Oshiro $^{1,4}$ and Yukihiro Yamaguchi ${ }^{1,4}$
}

\begin{abstract}
A 77-year-old man had undergone left-lobe liver resection and a choledochojejunostomy six years previously, and thereafter he suffered from a postoperative relapse of cholangitis. He was admitted to our hospital due to liver abscesses and bacteremia caused by multidrug-resistant Pseudomonas aeruginosa. Empirical treatment with piperacillin/tazobactam was started, and the patient initially recovered. However, he developed a second case of sepsis caused by piperacillin/tazobactam-resistant $P$. aeruginosa bacteremia originating from a new liver abscess. We changed the piperacillin/tazobactam to colistin and flomoxef and continued the two antibiotics for one month. During the antibiotic therapy, the patient successfully underwent bile duct stent placement.
\end{abstract}

Key words: multidrug-resistant Pseudomonas aeruginosa, liver abscess, colistin, colistimethate sodium, nephrotoxicity

(Intern Med 52: 1407-1412, 2013)

(DOI: 10.2169/internalmedicine.52.9296)

\section{Introduction}

Pseudomonas aeruginosa is an uncommon cause of liver abscesses. Chen et al. reported that only $1.86 \%(20 / 1,076$ cases) of liver abscess patients are infected with $P$. aeruginosa (1). In past case reports (2-10), only one patient with a multidrug-resistant Pseudomonas aeruginosa (MDRP) liver abscess patient has been described (10).

MDRP infection of any site is difficult to treat, due to restrictions on effective drugs, such as colistin. Colistin was developed in Japan in the 1940s. Early reports $(11,12)$ described common adverse effects of colistin, including nephrotoxicity and neurotoxicity. Due to these adverse events, the production of colistin was ceased in Japan in the 1990s. However, colistin has shown clinical effectiveness against multidrug-resistant Gram-negative organisms.
Many reports concerning MDRP infection and colistin have recently been published; however the use of colistin to treat a liver abscess caused by MDRP has not been described. We herein report a case of a liver abscess caused by MDRP and provide a review of the pertinent literature.

\section{Case Report}

This case involved a 77-year-old man who had undergone left lobe liver resection and choledochojejunostomy for early stage cholangiocarcinoma in September 2005. He had been treated for acute cholangitis three times in the prior six months. During his first cholangitis episode in March 2011, a blood culture was positive for three species: Citrobacter koseri, Enterococcus casseliflavus and P. aeruginosa. During the second episode in April 2011, Escherichia coli was detected on a blood culture. At the occasion of the third epi-

${ }^{1}$ Division of Infectious Diseases, Kenwakai Otemachi Hospital, Japan, ${ }^{2}$ Poison Center, Kenwakai Otemachi Hospital, Japan, ${ }^{3}$ Division of Surgery, Kenwakai Otemachi Hospital, Japan and ${ }^{4}$ Infection Control Team, Kenwakai Otemachi Hospital, Japan

Received for publication November 8, 2012; Accepted for publication February 11, 2013

Correspondence to Dr. Kenichiro Yaita, kyaita81@gmail.com 

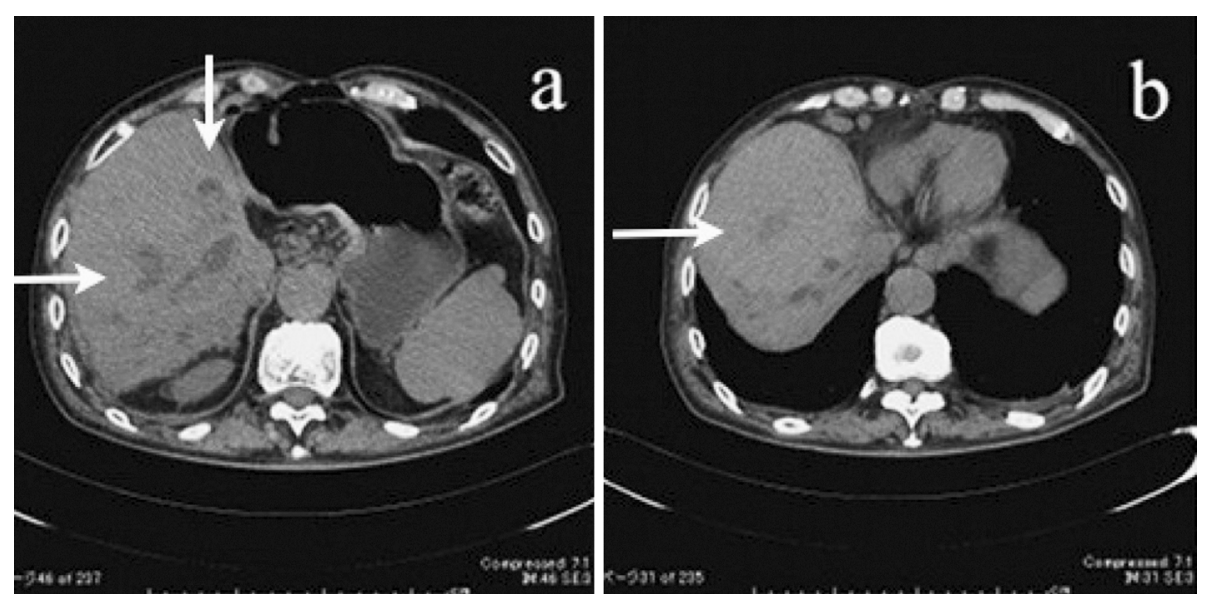

Figure 1. (a) Pseudomonas liver abscesses (S4: $\phi 20 \mathrm{~mm}, \mathrm{S8}: \phi 30 \mathrm{~mm}$ ) (arrows) detected on the day of admission. (b) A new abscess (S4/8: $\phi 40 \mathrm{~mm}$ ) (arrow) confirmed during piperacillin/tazobactam treatment.

Table 1. Antibiotic Resistance Profile of Two Strains of $P$. aeruginosa

\begin{tabular}{lcccc}
\hline & \multicolumn{3}{l}{ Strain $1(\mathrm{MDRP})$} & Strain 2 \\
Antibiotics & MIC $(\mu \mathrm{g} / \mathrm{mL})$ & $\mathrm{MIC}(\mu \mathrm{g} / \mathrm{mL})$ \\
\hline Piperacillin & $<64$ & $\mathrm{~S}$ & $<64$ & $\mathrm{~S}$ \\
Cefoperazone & $>32$ & $\mathrm{R}$ & $>32$ & $\mathrm{R}$ \\
Ceftazidime & $>16$ & $\mathrm{R}$ & $>16$ & $\mathrm{R}$ \\
Cefepime & $>16$ & $\mathrm{R}$ & $>16$ & $\mathrm{R}$ \\
Imipenem/Cilastatin & $>8$ & $\mathrm{R}$ & $>8$ & $\mathrm{R}$ \\
Meropenem & $>8$ & $\mathrm{R}$ & $>8$ & $\mathrm{R}$ \\
Aztreonam & $=16$ & $\mathrm{I}$ & $>16$ & $\mathrm{R}$ \\
Gentamicin & $>8$ & $\mathrm{R}$ & $>8$ & $\mathrm{R}$ \\
Amikacin & $>32$ & $\mathrm{R}$ & $=32$ & $\mathrm{I}$ \\
Levofloxacin & $>4$ & $\mathrm{R}$ & $>4$ & $\mathrm{R}$ \\
Ciprofloxacin & $>2$ & $\mathrm{R}$ & $>2$ & $\mathrm{R}$ \\
Colistin & $\mathrm{N} / \mathrm{A}$ & $\mathrm{S}$ & - & - \\
\hline
\end{tabular}

MDRP: multi-drug resistant pseudomonas aeruginosa, MIC: minimum inhibitory concentration

sode, no blood cultures were drawn. The patient had no other past medical history. His height was $168.5 \mathrm{~cm}$ and his body weight was $57.0 \mathrm{~kg}$.

He was admitted to Kenwakai Otemachi Hospital with moderate chills and fever in June 2011. His vital signs in the emergency room were as follows: a blood pressure of $150 / 90 \mathrm{mmHg}$, a heart rate of 70 beats/min, a respiratory rate of 25 breaths $/ \mathrm{min}$ and a body temperature of $39.2^{\circ} \mathrm{C}$. He had no abdominal symptoms (pain, nausea, diarrhea) and no abdominal tenderness. However, the values of laboratory tests were compatible with biliary inflammation: a white blood cell count of $8,400 / \mu \mathrm{L}$, a c-reactive protein level of $15.5 \mathrm{mg} / \mathrm{dL}$, a total bilirubin level of $5.7 \mathrm{mg} / \mathrm{dL}$, a direct bilirubin level of $2.3 \mathrm{mg} / \mathrm{dL}$, an aspartate aminotransferase level of $55 \mathrm{IU} / \mathrm{L}$, an alanine transaminase level of $63 \mathrm{IU} / \mathrm{L}$, an alkaline phosphatase level of $389 \mathrm{IU} / \mathrm{L}$ and a $\gamma$ glutamyltranspeptidase level of 117 IU/L. Abdominal computed tomography (CT) confirmed the presence of lowdensity areas (S4: $\phi 20 \mathrm{~mm}, \mathrm{~S} 8: \phi 30 \mathrm{~mm}$ ) in the liver
(Fig. 1a) that had not been detectable four months earlier. We diagnosed the patient with liver abscesses and initiated treatment with piperacillin/tazobactam at a dose of $4.5 \mathrm{~g}$ administered intravenously every six hours on the day of admission.

The patient became afebrile two days later, while a blood culture drawn on the day of admission was found to be positive for two strains of $P$. aeruginosa. One was MDRP (strain 1: meeting the definition of MDRP in Japan) and the other (strain 2: not meeting the definition of MDRP in Japan) was a strain resistant to most antipseudomonal drugs, including meropenem and ciprofloxacin. The susceptibility results and the minimum inhibitory concentration (MIC) of the two strains are described in Table 1. MDRP (strain 1) is only sensitive to piperacillin ( $\mathrm{MIC}<64 \mu \mathrm{g} / \mathrm{mL}$ ) and is resistant to meropenem (MIC $>8 \mu \mathrm{g} / \mathrm{mL}$ ), ciprofloxacin (MIC $>2$ $\mu \mathrm{g} / \mathrm{mL}$ ) and amikacin (MIC $>32 \mu \mathrm{g} / \mathrm{mL}$ ). A routine susceptibility test was performed using the Microscan WalkAway 96 Plus system (Siemens Japan K.K., Tokyo, Japan). The susceptibility of MDRP (strain 1) to colistin was assessed using the Kirby-Bauer method with Sensi-Disc ${ }^{\mathrm{TM}}$ Susceptibility Test Discs (Nippon Becton Dickinson Company, Tokyo, Japan). Later, a disk-diffusion screening test using sodium mercaptoacetic acid (metallo- $\beta$-lactamase SMA "EIKEN"; Eiken Cheminal Co., Ltd., Tokyo, Japan) showed that the MDRP strain produced metallo- $\beta$-lactamase. We also used a Break-point Checkerboard Plate (BC Plate "EIKEN"; Eiken Cheminal Co., Ltd.) (13); however, no synergic effects were observed for any combination of antibiotics.

On the 6th day, the patient underwent percutaneous transhepatic abscess drainage. On the 8th day, however, he again developed a fever $\left(39.4^{\circ} \mathrm{C}\right)$ with shaking chills. On the 10th day, the blood culture became positive for Gramnegative bacilli, and abdominal CT revealed a new abscess (S4/8: $\phi 40 \mathrm{~mm}$ ) in the liver (Fig. 1b). This second case of sepsis was caused by piperacillin/tazobactam-resistant $P$. aeruginosa bacteremia originating from the new abscess. This strain was found to be resistant to meropenem (MIC > 

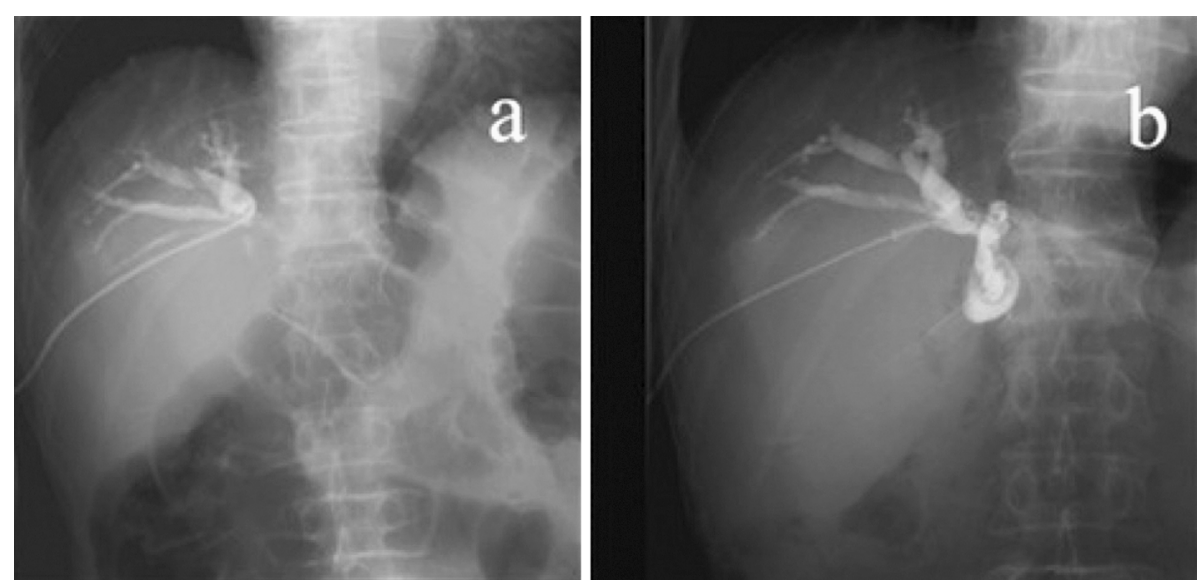

Figure 2. (a) Percutaneous transhepatic cholangiography revealed total obstruction of the biliary tract. (b) Successful placement of a metallic stent in the obstructive site in the biliary tract.

$8 \mu \mathrm{g} / \mathrm{mL}$ ) and ciprofloxacin (MIC $>2 \mu \mathrm{g} / \mathrm{mL}$ ) and intermittently resistant to amikacin (MIC $=8 \mu \mathrm{g} / \mathrm{mL}$ ).

Based on discussions held between infectious disease physicians, gastroenterological surgeons, the patient and his family, we decided to use colistin to treat the second case of sepsis. This decision was approved by the ethics committee of our hospital, as colistin was not available in Japan. On the 11th day, we initiated treatment with colistimethate sodium (colistin) (Coly-Mycin M Parenteral; JHP Pharmaceuticals, New Jersey, USA) at a dose of $150 \mathrm{mg}$ administered intravenously every 12 hours (serum creatinine (SCr): 0.73 $\mathrm{mg} / \mathrm{dL}$, estimated glomerular filtration rate (eGFR): $79 \mathrm{~mL} /$ $\mathrm{min})$. On the 12th day, the patient underwent percutaneous transhepatic cholangiodrainage, in which the operative findings revealed a bilioenteric anastomotic stricture (Fig. 2a). The drainage culture was positive for MDRP. On the 14th day, in addition to the administration of colistin, we changed the piperacillin/tazobactam regimen to flomoxef administered at a dose of $1 \mathrm{~g}$ intravenously every eight hours for anaerobic coverage; however the SCr level gradually increased. By the 18th day, the SCr level was $1.27 \mathrm{mg} / \mathrm{dL}$ and the eGFR was $43 \mathrm{~mL} / \mathrm{min}$. We reduced the doses of colistin and flomoxef (colistin: $75 \mathrm{mg}$ administered intravenously every 12 hours, flomoxef: $1 \mathrm{~g}$ administered intravenously every 12 hours). On the 34th day, a metallic stent was placed in the total obstructive site of the biliary tract (Fig. 2b). Since follow-up CT confirmed that the abscess had disappeared on the 38th day, we discontinued the administration of colistin after 28 days. At that point, the $\mathrm{SCr}$ level was $1.69 \mathrm{mg} / \mathrm{dL}$ and the eGFR was $31 \mathrm{~mL} / \mathrm{min}$, on the final day of colistin therapy. On the 45th day, the patient was discharged from the hospital. His SCr level did not recovered completely following colistin therapy. He has remained asymptomatic for approximately one year. His clinical course is presented in Fig. 3.

In our poison center, we measured the serum colistin concentrations in this case, retrospectively. We used a method primarily in reference to Ratjen's report (14). Blood samples (colistin administration days $0,2,3,4,7,9$ and 10) were stored at $-50^{\circ} \mathrm{C}$. The samples were collected prior to colistin administration (trough concentration). $20 \mu \mathrm{L}$ of aqueous internal standard solution $(10 \mu \mathrm{g} / \mathrm{mL}$ of netilmicin sulphate) and $25 \mu \mathrm{L}$ of methanol/10\% trifluoroacetic acid (1/1) were added to $150 \mu \mathrm{L}$ of serum. After shaking the samples for 30 seconds on a vortex shaker followed by centrifugation $(12,000 \mathrm{rpm}, 3 \mathrm{~min})$, we transferred the supernatant to a polypropylene tube for derivatization. Sep-Pak C18 cartridges $(1 \mathrm{cc} / 50 \mathrm{mg}$, Waters $)$ were prepared using $1 \mathrm{~mL}$ of methanol and $1 \mathrm{~mL}$ of $1 \%$ sodium hydrogen carbonate buffer ( $\mathrm{pH} 10)$, after which the supernatant was added along with another $1 \mathrm{~mL}$ of the carbonate buffer solution. Next, $30 \mu \mathrm{L}$ of $50 \mathrm{mM}$ 9-fluorenylmethyl chloroformate was added and left to react for 15 minutes. After washing with 2 $\mathrm{mL}$ of $70 \%$ acetonitrile, we eluted the samples with $900 \mu \mathrm{L}$ of acetone. $600 \mu \mathrm{L}$ of $0.2 \mathrm{M}$ boric acid solution was added to the eluates. High-performance liquid chromatography was carried out by injecting $10-\mu \mathrm{L}$ aliquots of the processed samples onto a Develosil ODS-UG-5 column $(150 \times 4.6 \mathrm{~mm}$, $5 \mu \mathrm{m})$. The column temperature was maintained at $35^{\circ} \mathrm{C}$. The samples were eluted with a mobile phase of acetonitrile/tetrahydrofuran/water (46/32/22). The flow rate was 1 $\mathrm{mL} / \mathrm{min}$. A fluorescence detector (excitation wavelength, 260 $\mathrm{nm}$; emission wavelength, $315 \mathrm{~nm}$ ) was used for detection. The results of this analysis are described in Fig. 3.

\section{Discussion}

P. aeruginosa is an uncommon cause of liver abscesses. Among all pyogenic liver abscesses, the prevalence of $P$. aeruginosa liver abscesses (PALA) has been reported to be 5.3-7.6\% (15, 16). Chen et al. reported the most recent case series in which 20 PALA patients $(1.86 \%)$ were found among a total of 1,076 pyogenic liver abscess patients followed for seven years (1). In that report, 17 patients (85\%) had hepatobiliary comorbidities, such as common biliary duct stones (three cases) and pancreatic head tumors (two cases). In all, 13 patients (65\%) had undergone a previous procedures, including endoscopic retrograde cholangiopan- 


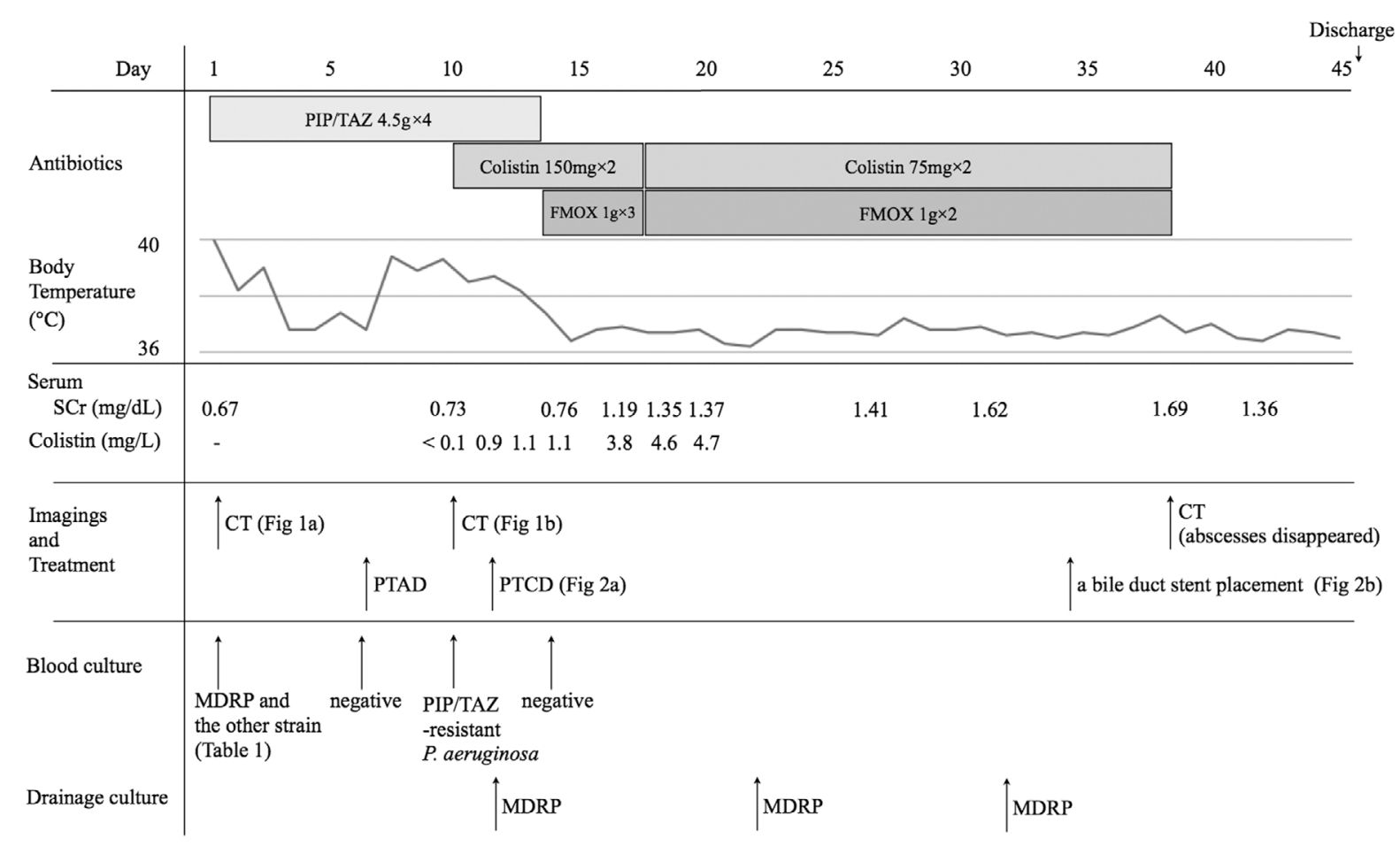

Figure 3. Clinical course. CT: computed tomography, FMOX: flomoxef, MDRP: multidrug-resistant Pseudomonas aeruginosa, PIP/TAZ: piperacillin/tazobactam, PTAD: percutaneous transhepatic abscess drainage, PTCD: percutaneous transhepatic cholangiodrainage, $\mathrm{SCr}$ : serum creatinine

creatography (five cases). The researchers concluded that hepatobiliary comorbidities/intra-abdominal procedures are risk factors of PALA. We searched all published PALA case reports written in English and summarized the features of these cases (2-10) in Table 2. As with Chen's study, most patients had hepatobiliary comorbidities and/or had undergone intra-abdominal procedures. Only two patients $(6,10)$ were previously healthy. These results are compatible with our patient's situation. In addition, only one previous case (10) has so far been described as an MDRP liver abscess treated with imipenem plus amikacin. As far as we could ascertain, the present study marks the first case report of an MDRP liver abscess treated with colistin.

The definition of MDRP is unclear and arbitrary (17); various definitions (18) are used in the prioir literature. In Japan, the definition of MDRP is that of a strain that is resistant to imipenem, amikacin and ciprofloxacin. We refer to the Japanese criteria to describe this case.

When treating MDRP infection, there is a limited choice of antibiotics, including colistin. This is a venerable drug that was discovered in the 1940s in Japan (19) and was replaced by other antibiotics due to adverse effects, such as nephrotoxicity and neurotoxicity $(11,12)$. In Japan, colistin currently is not officially approved (October 2012); however, it is expected to gain approval in the near future.

Although colistin has been in existence for a long time, many aspects of its effects remain to be clarified. The spectrum of colistin is already known; it is active against most Gram-negative bacilli, including multidrug-resistant Pseudomonas aeruginosa (20). However, the optimal dose and pharmacodynamics of colistin have not been well studied. The pharmacodynamics of colistin appear to be concentration-dependent (20), and the area under the curve/ minimum inhibitory concentration is the most effective parameter (21). However, studies to detect optimum dosing strategies are insufficient. In a recent report (22), a lower colistin dose treatment was found to be associated with higher mortality (3 million IU/day; 38.6\%, 6 million IU/day; $27.8 \%$ vs. 9 million IU/day; 21.7\%); therefore, a higher dose of colistin is recommended. However, since manufacturers recommend a dose of $2.5-5 \mathrm{mg} / \mathrm{kg} / \mathrm{day}(1 \mathrm{mg}=12,500$ IU) and we had no experience with colistin, we first used $300 \mathrm{mg} /$ day of colistin (=3.75 million IU/day).

Nephrotoxicity is a common adverse effect of colistin. In an early report (12), nephrotoxicity occurred in $20.2 \%$ of cases. However, recently, the rate of nephrotoxicity has been reported to be lower. Falagas et al. (22) reported the findings of a retrospective cohort study of 258 patients treated with colistin, and found that 26 patients (10\%) developed nephrotoxicity. In another study (23), chronic renal insufficiency, diabetes mellitus and aminoglycoside use were suggested to be risk factors for nephrotoxicity (odds ratios: 11.3, 5.1 and 5, respectively). Nephrotoxicity has also been documented during long periods of administration (more than four weeks) of colistin (24). Although the administration dose used in that study was relatively low $(4.4 \pm 2.1$ million IU $=352 \pm 168 \mathrm{mg} /$ day) and only a small number of cases (19 cases) were analyzed, the median serum creatinine levels increased by only $0.25 \mathrm{mg} / \mathrm{dL}$ (24). This difference in findings between older and more recent reports may be re- 
Table 2. Previous Case Reports of Pseudomonas aeruginosa Liver Abscesses (Published in English)

\begin{tabular}{|c|c|c|c|c|c|c|c|}
\hline Reference & $\begin{array}{l}\text { Age/Sex } \\
\text { (year) }\end{array}$ & $\begin{array}{l}\text { Community-acquired/ } \\
\text { Nosocomial }\end{array}$ & Co-morbidities & Preceding procedure & $\begin{array}{l}\text { Definitive antibiotic } \\
\text { therapy }\end{array}$ & Surgical therapy & Outcome \\
\hline $\begin{array}{l}\text { Grieco et al. } \\
\text { (2) }\end{array}$ & 32/Male & Nosocomial & acute gastrointestinal bleeding & $\begin{array}{l}\text { subtotal gastrectomy, } \\
\text { gastrojejunomy }\end{array}$ & GM, Polymixin B & Surgical drainage & Survive \\
\hline $\begin{array}{l}\text { Wallack et al. } \\
\text { (3) }\end{array}$ & 53/Male & Community-acquired & sigmoid diverticulitis & No & $\begin{array}{l}\text { GM, carbenicillin, } \\
\text { cephalothin }\end{array}$ & Surgical drainage & Survive \\
\hline $\begin{array}{l}\text { Davion et al. } \\
\text { (4) }\end{array}$ & 44/Male & Community-acquired & No & ERCP & carbenicillin & PTAD & Survive \\
\hline $\begin{array}{l}\text { Korvick et al. } \\
\text { (5) }\end{array}$ & $41 /-$ & - & rejection of prior liver & liver transplant & IPM, TOB, RFP & - & Survive \\
\hline $\begin{array}{l}\text { Korvick et al. } \\
\text { (5) }\end{array}$ & $13 /-$ & - & failure of 1st transplant & liver transplant & CPFX & - & Survive \\
\hline $\begin{array}{l}\text { Lo et al. } \\
\text { (6) }\end{array}$ & 1/ Male & Community-acquired & No & No & CAZ, GM & Aspiration only & Survive \\
\hline $\begin{array}{l}\text { Katsinelos et al. } \\
\text { (7) }\end{array}$ & 32/Male & Community-acquired & sphincter of Oddi dysfunction & ERCP & $\mathrm{PIP}, \mathrm{AMK} \rightarrow \mathrm{CPFX}$ & No & Survive \\
\hline $\begin{array}{l}\text { Goldani et al. } \\
\text { (8) }\end{array}$ & 43/Male & Community-acquired & $\begin{array}{l}\text { chronic schistosomiasis, Previous } \\
\text { P. aeruginosa liver abscess }\end{array}$ & $\begin{array}{l}\text { laparoscopic } \\
\text { cholecystectomy }\end{array}$ & $\mathrm{CPFX} \rightarrow \mathrm{CAZ}$ & $\begin{array}{l}\text { PTAD, } \\
\text { Surgical resection }\end{array}$ & Survive \\
\hline $\begin{array}{l}\text { Losanoff et al. } \\
\text { (9) }\end{array}$ & 22/Male & Nosocomial & liver transplant at age 2 years & $\begin{array}{l}\text { Gun-shot injury of liver and } \\
\text { liver repairing operation }\end{array}$ & - & PTAD & Survive \\
\hline $\begin{array}{l}\text { Ulug at al. } \\
(10)\end{array}$ & 28/Male & Community-acquired & No & No & IPM, AMK & PTAD & Survive \\
\hline
\end{tabular}

AMK: amikacin, CAZ: ceftazidime: CPFX: ciprofloxacin, ERCP: endoscopic retrograde cholangiopancreatography, GM: gentamicin, IPM: imipenem, PIP: piperacillin, PTAD: percutaneous transhepatic abscess drainage, RFP: rifampin, TOB: tobramycin

lated to purification of colistin, improvements in the support available for severe patients, differences in dosage or avoidance of the coadministration of other agents influencing the renal function $(20,24)$. In our case, the serum creatinine level and trough concentration of colistin were significantly increased during the course of treatment (Fig. 3) and we did not administer other drugs that could cause renal function disorders. We speculate that it is possible that the serum creatinine level did not reflect the patient's true renal function. His body mass index was 20.1 and his muscular volume was low in appearance. When renal function problems are exacerbated following the administration of colistin, early discontinuation is necessary (25). However, we had no choice but to continue the colistin administration due to the resistance of MDRP to other antibiotics. Renal dose adjustment may therefore help to protect against the development of further renal function disorders.

Based on the facts of this case and those found in our literature review, we propose the following three points. First, clinicians should remember that hepatobiliary comorbidities and/or intra-abdominal procedures are risk factors for the development of $P$. aeruginosa liver abscesses. Second, colistin is effective against PALA caused by MDRP. Third, in this case, we were able to successfully administer colistin for a 28-days period through renal dose adjustment.

\section{The authors state that they have no Conflict of Interest (COI).}

\section{Acknowledgement}

The authors thank the Infection Control Team of the University of Occupational and Environmental Health, Japan for their help in our effective and safe use of colistin.

\section{References}

1. Chen WH, Chiu $\mathrm{CH}$, Huang $\mathrm{CH}$, et al. Pyogenic liver abscess caused by Pseudomonas aeruginosa: clinical analysis of 20 cases. Scand J Infect Dis 43: 877-882, 2011.

2. Grieco MH, McCray RS, Nay HR. Gentamicin therapy for liver abscesses due to $P$. aeruginosa and $S$. marcescens. NY State J Med 73: 1108-1110, 1973.

3. Wallack MK, Brown AS, Austrian R, Fitts WT. Pyogenic liver abscess secondary to asymptomatic sigmoid diverticulitis. Ann Surg 184: 241-243, 1976.

4. Davion T, Braillon A, Delamarre J, Delcenserie R, Joly JP, Capron JP. Pseudomonas aeruginosa liver abscesses following endoscopic retrograde cholangiography. Report of a case without biliary tract disease. Dig Dis Sci 32: 1044-1046, 1987.

5. Korvick JA, Marsh JW, Starzl TE, Yu VL. Pseudomonas aeruginosa bacteremia in patients undergoing liver transplantation: an emerging problem. Surgery 109: 62-68, 1991.

6. Lo WT, Wang CC, Hsu ML, Chu ML. Pyogenic liver abscess caused by Pseudomonas aeruginosa in a previously healthy child: report of one case. Acta Paediatr Taiwan 41: 98-100, 2000.

7. Katsinelos P, Dimiropoulos S, Katsiba D, et al. Pseudomonas aeruginosa liver abscesses after diagnostic endoscopic retrograde cholangiography in two patients with sphincter of Oddi dysfunction type 2. Surg Endosc 16: 1638, 2002.

8. Goldani LZ, dos Santos RP, Sugar AM. Pyogenic liver abscess in patients with schistosomiasis mansoni. Trans R Soc Trop Med Hyg 99: 932-936, 2005.

9. Losanoff JE, Millis JM. Giant necrotizing abscess of a liver transplant after gunshot injury. Transplantation 84: 1373-1374, 2007.

10. Ulug M, Gedik E, Girgin S, Celen MK, Ayaz C. Pyogenic liver abscess caused by community-acquired multidrug resistance Pseudomonas aeruginosa. Braz J Infect Dis 14: 218, 2010.

11. Brown JM, Dorman DC, Roy LP. Acute renal failure due to overdosage of colistin. Med J Aust 2: 923-924, 1970. 
12. Koch-Weser J, Sidel VW, Federman EB, Kanarek P, Finer DC, Eaton AE. Adverse effects of sodium colistimethate. Manifestations and specific reaction rates during 317 courses of therapy. Ann Intern Med 72: 857-868, 1970.

13. Tateda $K$, Ishii $Y$, Matsumoto $T$, Yamaguchi $K$. 'Break-point Checkerboard Plate' for screening of appropriate antibiotic combinations against multidrug-resistant Pseudomonas aeruginosa. Scand J Infect Dis 38: 268-272, 2006.

14. Ratjen F, Rietschel E, Kasel D, et al. Pharmacokinetics of inhaled colistin in patients with cystic fibrosis. J Antimicrob Chemother 57: 306-311, 2006.

15. Ribaudo JM, Ochsner A. Intrahepatic abscesses: amebic and pyogenic. Am J Surg 125: 570-574, 1973.

16. Silver S, Weinstein A, Cooperman A. Changes in the pathogenesis and detection of intrahepatic abscess. Am J Surg 137: 608-610, 1979.

17. Paterson DL. The epidemiological profile of infections with multidrug-resistant Pseudomonas aeruginosa and Acinetobacter species. Clin Infect Dis 43 (Suppl 2): S43-S48, 2006.

18. Falagas ME, Koletsi PK, Bliziotis IA. The diversity of definitions of multidrug-resistant (MDR) and pandrug-resistant (PDR) Acinetobacter baumannii and Pseudomonas aeruginosa. J Med Microbiol 55: 1619-1629, 2006.
19. Koyama Y, Kurosasa A, Tsuchiya A, Takakuta K. A new antibiotic "colistin" produced by spore-forming soil bacteria. J Antibiot (Tokyo) 3: 457-458, 1950 (in Japanese).

20. Falagas ME, Kasiakou SK. Colistin: the revival of polymyxins for the management of multidrug-resistant gram-negative bacterial infections. Clin Infect Dis 40: 1333-1341, 2005.

21. Bergen PJ, Bulitta JB, Forrest A, Tsuji BT, Li J, Nation RL. Pharmacokinetic/pharmacodynamic investigation of colistin against Pseudomonas aeruginosa using an in vitro model. Antimicrob Agents Chemother 54: 3783-3789, 2010.

22. Falagas ME, Rafailidis PI, Ioannidou E, et al. Colistin therapy for microbiologically documented multidrug-resistant Gram-negative bacterial infections: a retrospective cohort study of 258 patients. Int J Antimicrob Agents 35: 194-199, 2010.

23. Montero M, Horcajada JP, Sorlí L, et al. Effectiveness and safety of colistin for the treatment of multidrug-resistant Pseudomonas aeruginosa infections. Infection 37: 461-465, 2009.

24. Falagas ME, Rizos M, Bliziotis IA, Rellos K, Kasiakou SK, Michalopoulos A. Toxicity after prolonged (more than four weeks) administration of intravenous colistin. BMC Infect Dis 5: 1, 2005.

25. Falagas ME, Kasiakou SK. Toxicity of polymyxins: a systematic review of the evidence from old and recent studies. Crit Care 10: R27, 2006.

(C) 2013 The Japanese Society of Internal Medicine http://www.naika.or.jp/imonline/index.html 